\title{
Clinical Presentation, Management and Outcome in Diverticular Colovesical Fistulas - Our Experience
}

\author{
Gaurav Kalra ${ }^{1}$, Rajeev Thekke Puthalath², Suraj Hegde ${ }^{3}$, Narendra Pai ${ }^{4}$, Amit Kumar ${ }^{5}$
}

\begin{abstract}
${ }^{1}$ Department of Urology, K.S. Hegde Medical Academy, NITTE (Deemed to Be University), Mangalore, Karnataka, India. ${ }^{2}$ Department of Urology, K.S. Hegde Medical Academy, NITTE (Deemed to Be University), Mangalore, Karnataka, India. ${ }^{3}$ Department of Urology, K.S. Hegde Medical Academy, NITTE (Deemed to Be University), Mangalore, Karnataka, India. ${ }^{4}$ Department of Urology, K.S. Hegde Medical Academy, NITTE (Deemed to Be University), Mangalore, Karnataka, India. ${ }^{5}$ Department of Urology, K.S. Hegde Medical Academy, NITTE (Deemed to Be University), Mangalore, Karnataka, India.
\end{abstract}

\section{ABSTRACT}

\section{BACKGROUND}

Colovesical fistula (CVF) is an abnormal communication between the urinary bladder and the large intestine, usually sigmoid colon. Diverticulitis is the most common cause of CVF in most of the western studies, accounting for approximately $70 \%$ of cases. Diverticular CVF is uncommon in Asia. This case series shares the experience of six cases of diverticular CVF in Indian population.

\section{METHODS}

Medical records of six patients with diverticular colovesical fistulas during the period January 2016 - August 2019 were reviewed with regard to symptoms, diagnostic investigations, and management. Various aspects of the disease were analysed to determine the common features of colovesical fistula in our population.

\section{RESULTS}

All patients with diverticular colovesical fistula were presented with urinary symptoms and none were aware about their existing colonic diverticulosis. Five out of the six cases presented with pneumaturia. Contrast enhanced computed tomography (CECT) abdomen detected sigmoid diverticulosis with vesical fistula in all cases. The most common site of fistula found on cystoscopy was on the left superolateral wall of bladder. All cases were operated as a single stage procedure including fistula repair, colonic resection, omental interposition with no temporary colostomy which provided an excellent surgical cure.

\section{CONCLUSIONS}

Colovesical fistula secondary to diverticular disease has shown a rising incidence and can be effectively managed by a multidisciplinary team. It requires prompt diagnosis, adequate preoperative evaluation, perioperative care including bowel preparation, nutritional supplementation, appropriate antibiotics, and meticulous surgical skills allowing an elective one-stage approach.

\section{KEY WORDS}

Colovesical Fistula, Diverticular Disease, Diverticular Colovesical Fistula, Pneumaturia, Single Stage Repair
Corresponding Author: Dr. Rajeev Thekke Puthalath, Professor and HOD, Department of Urology, K.S. Hegde Medical Academy, NITTE (Deemed to be University) Post Nithyananda Nagar, Deralakatte, Mangalore-575018, Karnataka, India. E-mail: rajeevtp@yahoo.com

DOI: $10.14260 / \mathrm{jemds} / 2020 / 466$

How to Cite This Article:

Kalra G, Puthalath RT, Hegde S, et al. Clinical presentation, management and outcome in diverticular colovesical fistulasour experience. J. Evolution Med. Dent. Sci. 2020;9(30):2139-2143, DOI: $10.14260 /$ jemds/2020/466

Submission 22-04-2020,

Peer Review 17-06-2020,

Acceptance 24-06-2020,

Published 27-07-2020.

Copyright (C) 2020 JEMDS. This is an open access article distributed under Creative Commons Attribution License [Attribution 4.0 International (CC BY 4.0)] 


\section{BACKGROUND}

Colovesical fistula (CVF) is an abnormal communication between urinary bladder and large intestine, usually the sigmoid colon. Diverticulitis of colon is the most common cause of colovesical fistulae in most of the western case series, accounting for approximately $70 \%$ of cases. ${ }^{1}$ Other causes of CVF are malignancies, Crohn's disease, trauma, foreign bodies, and perforated appendicular abscess. Interestingly, only approximate $2 \%$ of patients with diverticulitis can experience a colovesical fistula as a complication of their disease. Diverticular disease of colon is a disease of western civilization with high prevalence seen in United States, Europe and Australia. Meanwhile in Asia, diverticular disease is much less commonly diagnosed and if present, nearly $70 \%$ of cases have diverticula located in the right colon. ${ }^{2}$ The peak incidence of diverticular disease of colon happens to be in 65 to 75 years of age. ${ }^{3}$

Diverticula are abnormal outpouchings or sacs of the colon wall that occur most commonly because of interactions of high intraluminal pressures, altered colon motility, alterations in colonic structure, faecal stasis and diets low in fibre. Lowvolume stools noted in low-fibre diets prevalent in western societies incur higher intraluminal pressures during colonic muscle contraction. Diverticular disease of the colon is known for its varied spectrum of presentation, and is divided into uncomplicated diverticulosis and complicated diverticulosis. Complicated diverticulosis further divided into uncomplicated diverticulitis which presents as localized phlegmon and complicated diverticulitis having abscess, free perforation with peritonitis, fistula, or obstruction and bleeding.

Faecal stasis within diverticula is often suggested as a possible inciting factor in acute diverticulitis. Smoking due to nicotine decreases the formation of collagen, increases colonic motility and intraluminal pressure, and impairs blood flow to the colonic mucosa, which may then lead to diverticular disease. Obesity, increasing waist circumference and waist-tohip ratio were also associated with the development of diverticulitis and diverticular bleeding. Chronic use of Corticosteroids may increase the risk of perforation in diverticulitis, perhaps due to the reduction in collagen turnover that reduces colon wall integrity.

The average age of presentation of a colovesical fistula is 61 years. ${ }^{4}$ But the exact age group of occurrences of diverticular CVF is not available in the literature. Clinical presence of pneumaturia and recurrent urinary tract infections (UTI) or cystitis not responding to antibiotic therapy may suggest a colovesical fistula and need evaluation. ${ }^{5}$ Clinical examination of a suspected CVF patient will not yield any clue for the diagnosis. Hence confirmation of the condition is mostly based on the findings from imaging modalities. There are reports of usefulness of abdominal and pelvic ultrasonography for the diagnosis of colovesical fistulae with a characteristic "beak" sign. The investigation of choice for the diagnosis of diverticular colovesical fistula is CT scan of abdomen which not only confirm the diagnosis but also can demonstrate the nature of diverticular disease and its extent. Cystoscopy is an invasive investigation that can detect an abnormality in more than $90 \%$ of cases. The study by Pollard et $\mathrm{al}^{4}$ showed that dome of bladder was the most common location of fistula.
There is good consensus internationally about recommendations for resection for patients with diverticular CVF. Once the decision for surgical management is made, the standard treatment is focused on identification and division of the fistula, resection of the involved portion of the colon, and bladder repair if necessary. While it has been demonstrated that excision of the involved colon (most commonly sigmoid) is necessary due to the high rate of recurrence with a simple division of the fistula without resection. ${ }^{4}$

Colovesical fistulae secondary to diverticular disease has shown a rising incidence, though diverticular disease is uncommon in Asia. This study shares the experience of treating six diverticular CVF cases in Indian population. This study puts in an effort to feature the clinical presentation, management and outcome of the diverticular colovesical fistulas.

\section{METHODS}

This is a retrospective study based of case series analysis conducted in the department of Urology at K.S. Hegde medical academy, Mangalore. The study period included January 2016 to August 2019. There were six cases of diverticular CVF surgically treated during this period. The data regarding the demographic details, clinical presentation, diagnostic methods, management and outcomes of the patient with diverticular CVF were collected.

Our institution mainly caters treatment of patients from coastal Karnataka and northern Kerala of south India. All of these six cases belong to the same region. CECT abdomen and pelvis with oral, intravenous and rectal and contrast was the diagnostic modality we selected in suspected CVF cases. Colonoscopy and cystoscopy were done in all cases prior to the definite management. Elective cystoscopy was performed to identify the site of fistula and to rule out malignancy. All cases were operated as a single stage operation. The intraoperative findings, the types of surgical procedures performed, and the post-operative course were acquired from the case files. The follow up records in terms of outcome and any complications were also analyzed.

\section{Statistical Analysis}

The data was collected, compiled and analysed. Data was entered into Microsoft Excel (Windows 7; Version 2007) and analyses were done using the Statistical Package for Social Sciences (SPSS) for Windows software (Version 22.0; SPSS Inc, Chicago). Descriptive statistics such as mean and standard deviation (SD) for continuous variables, frequencies and percentages were calculated for categorical Variables were determined.

\section{RESULTS}

All our patients with diverticular CVF series were male. The oldest patient was of 80 years and youngest being 45 years. The mean age of presentation was 61 years. The most common clinical presentation we noted was pneumaturia. The clinical 
presentations according to duration of symptoms were given in Table 1. Out of six patients two had concurrent diabetes and hypertension and one patient was a known case of bronchial asthma.

In all patients, routine blood investigations were done Renal function tests were within normal limits in all cases. Urine culture \& sensitivity showed a significant growth of organisms in all cases. Escherichia coli being the most common organism isolated in four cases and in two cases mixed flora was found. Ultrasound abdomen \& pelvis in all six patients found an irregular bladder wall thickening but was unremarkable otherwise.

\begin{tabular}{|cc|}
\hline Clinical Symptoms \& Duration & Number of Patients \\
Pneumaturia (6 month) & 5 \\
Recurrent UTI (6 month) & 3 \\
Fecaluria (2 month) & 1 \\
Lower abdomen pain (1 year) & 2 \\
Table 1. Clinical Symptoms \& Their Duration \\
\hline
\end{tabular}

\begin{tabular}{|cc|}
\hline Sl. No. & Cystoscopy Findings and Fistula Sites \\
Case 1 & Erythematous Irregular areas on left Superolateral wall \\
Case 2 & Fistula on right superolateral wall with mucus extruding. \\
Case 3 & Faecal material in urinary bladder. \\
Case 4 & Fistula on left lateral wall \\
Case 5 & Fistula on left superolateral wall with mucus extruding \\
Case 6 & Fistula on left superolateral wall with bullous edema \\
& Papillary projection at right anterior wall of bladder near dome. \\
& Table 2. Cystoscopy Findings \\
\hline
\end{tabular}

\begin{tabular}{|c|c|c|}
\hline Sl. No. & Bla & Bowel Ma \\
\hline Case 1 & $\begin{array}{l}\text { Excision of fistula as a bladder cuff } \\
\text { with Sigmoid colon. Bladder defect } \\
\text { closed in two layers }\end{array}$ & $\begin{array}{l}\text { Sigmoid colectomy followed by } \\
\text { descending colon to rectum } \\
\text { anastomosis }\end{array}$ \\
\hline Case 2 & Excision of fistula \& bladder repair & $\begin{array}{l}\text { Left hemicolecto } \\
\text { colo-colonic }\end{array}$ \\
\hline Case 3 & $\begin{array}{l}\text { Excision of fistulous tract with } \\
\text { surrounding } 1 \mathrm{~cm} \text { of bladder wall and } \\
\text { the bladder defect closed in } 2 \text { layers }\end{array}$ & $\begin{array}{r}\text { Sigmoid Resect } \\
\text { distal } 2 / 3 \text { of des } \\
\text { diverticula rese } \\
\text { anas }\end{array}$ \\
\hline Case 4 & $\begin{array}{r}\text { Partial } \\
\text { sigmoid colon a } \\
\text { and part of urin }\end{array}$ & $\begin{array}{c}\text { Sigmoid colon, entire descending } \\
\text { colon resected followed by colorectal } \\
\text { anastomosis }\end{array}$ \\
\hline Case 5 & $\begin{array}{c}\text { Partial cystectomy } \\
\text { Sigmoid diverticulum with hard } \\
\text { indurated lesion } 5^{*} 5 \mathrm{~cm} \text { fixed to } \\
\text { dome, lateral wall of bladder, lateral } \\
\text { pelvic wall \& external iliac vein }\end{array}$ & $\begin{array}{c}\text { The indurated mass dissected after } \\
\text { mobilizing sigmoid colon. Sigmoid } \\
\text { colectomy and end to end colonic } \\
\text { anastomosis done. }\end{array}$ \\
\hline Case 6 & Excision of fistula \& bladde & $\begin{array}{l}\text { Left hemicolectomy followed by colo- } \\
\text { colonic anastomosis }\end{array}$ \\
\hline \multicolumn{3}{|c|}{ Table 3. Operative Procedure } \\
\hline
\end{tabular}

CECT abdomen and pelvis revealed sigmoid diverticulosis, air in urinary bladder and bladder wall thickening with CVF in all cases. Gastroenterologist performed a colonoscopy, which revealed multiple sigmoid colon diverticula in all cases, with two had additional diverticula in descending colon. An elective cystoscopy was performed to confirm the diagnosis and the most common site of fistula was on the left superolateral wall of the bladder. There were few other significant findings on cystoscopy, which are given in Table 2 .

All cases were surgically treated by a single stage operation. Surgical team included specialists from urology and gastro surgery departments. The surgical procedure included an exploratory laparotomy through lower midline incision to confirm the diagnosis of colovesical fistula followed by an attempt to separate the bladder from the bowel. Operative procedure divided into two steps as bladder management and bowel management, which are summarised in Table 3.

We had mortality in our case series, as one patient expired on the second postoperative day due to pulmonary embolism. Other complications were minor wound dehiscence in one and the late recurrence of fistula seen in another one at follow up.
Histopathologic evaluation of all six specimens revealed diverticulitis with colovesical fistula. There was no evidence of malignancy or granuloma in these cases. The biopsy of urinary bladder specimen showed cystitis cystica in all cases.

\section{DISCUSSION}

There is sparse data of diverticular colovesical fistula from Indian population. Causes of colovesical fistula are diverticulitis in 65-75\%, malignancy in 10-15\%, Crohn's disease in 5-6\% and trauma, appendiceal abscess and foreign body in approximately $5 \%$ of cases. ${ }^{6}$

In literature the commonest symptoms of colovesical fistula is pneumaturia (52-77\%). Our study shows in $80 \%$ of patients presented with pneumaturia in CVF secondary to diverticular colonic disease. Interestingly none of our patients were diagnosed to have diverticular disease of the colon prior and had first time presented to urology department with symptoms. There has been significant delay in clinical diagnosis from the time of first presentation, due to the rarity of this entity. Patients themselves gave history of pneumaturia and recurrent UTI not responding to multiple antibiotics. A clinical suspicion of diverticular CVF was possible in five cases in view of pneumaturia.

Other symptoms of CVF are faecaluria (36-51\%), urinary tract infection symptoms like frequency, urgency, dysuria (44$45 \%)$, fever and chills (41\%), abdominal pain (25\%), nonspecific gastrointestinal symptoms (25\%) and hematuria (5$22 \%$ ). None of our patients presented with hematuria, but we had a one patient presented with fecaluria. It is difficult to suspect diverticular CVF as the diagnosis in patients with recurrent UTI or cystitis refractory to antibiotic therapy, unless the surgeon considers its possibility ${ }^{7}$. Gouverneur syndrome is characterized by vesicointestinal fistula with associated suprapubic pain, urinary frequency, dysuria and tenesmus. $^{8}$ In the literature a positive urine culture is reported in $78 \%$ of cases with $58 \%$ of these having a mixed growth. In our study, urine culture was positive in $66.6 \%$ patients with mixed flora in $33.3 \%$ patients.

Uncomplicated diverticulosis can be asymptomatic diverticulosis and symptomatic uncomplicated diverticular disease (SUDD). Whereas complicated diverticulosis further sub-divided into uncomplicated diverticulitis and complicated diverticulitis. Uncomplicated diverticulitis usually presents as localized phlegmon and complicated diverticulitis can have abscess, free perforation with peritonitis, fistula, or obstruction and bleeding.

Ultrasonography has been reported to be useful in the diagnosis of colovesical fistulae. A characteristic "beak" sign may be noted. ${ }^{9}$ In our case series ultrasound abdomen $\&$ pelvis revealed bladder wall thickening and chronic cystitis, but unable to suspect a fistula. Cross-sectional imaging, especially CECT abdomen and pelvis scanning, has become the imaging modality of choice. The triad of findings on CT that are suspicious for colovesical fistulae consist of (1) bladder wall thickening adjacent to a loop of thickened colon (2) air in the bladder (in the absence of previous lower urinary manipulation). (3) presence of colonic diverticula.10,11,12

All of our patients had these findings in CT, and hence we consider CECT abdomen is the most accurate imaging 
modality to diagnosis diverticular CVF. The sensitivity and specificity of this modality with diagnostic accuracy is as high as $90 \%$ to $100 \% \cdot{ }^{13,14,15}$ Air in the bladder on diagnostic imaging is suggestive of colovesical fistulae, false positives may be seen in recent instrumentation (catheterization, cystoscopy) and active UTI with a gas-forming organism. CECT abdomen and pelvis scanning should be done after the administration of oral contrast but before the administration of intravenous contrast to permit detection of barium within the bladder. Cystography and transrectal contrast studies (e.g. barium enema) are in general less likely to demonstrate the fistula. Colonoscopy is valuable in evaluating for colonic disease, such as malignancy, as a cause of the fistula; the preoperative knowledge of such a condition can considerably alter management decisions.

Cystoscopy has the highest yield in identifying a potential lesion, abnormality noted on endoscopic examination in more than $90 \%$ of cases. ${ }^{16}$ However, the findings on cystoscopy are often nonspecific and include localized erythema, papillary, or bullous change; a definitive diagnosis using cystoscopy can be made in only $35 \%$ to $46 \%$ of cases. Cystoscopy and biopsy of abnormal appearing tissue are indicated to evaluate for the possibility of a malignant fistula. In our study consistently we found location of the fistula on the left superolateral wall of bladder part near to sigmoid colon. The study by Pollard et $\mathrm{al}^{4}$ showed that showed dome of bladder was the most common location of fistula in $62 \%$ of cases.

Historically, some other tests in diagnosis of colovesical fistula were described which may not relevant now a days. Bourne test is described as a non-diagnostic barium enema, first voided urine is immediately centrifuged \& examined radiographically, radiodense particles in the urine are considered a positive test result. ${ }^{17}$ Vesicoenteric fistulae may be confirmed by the oral administration of activated charcoal, which in the setting of a fistula will appear in the urine as black particles, known as charcoal test. 18 This test provides no anatomic information regarding the location of the fistula. A group of urologists in Germany described Poppy seed test in which the patient is fed 1.25 ounces of poppy seeds with 12 ounces of fluid and the patient's urine is then collected for the next 48 hours and examined for poppy seeds. ${ }^{19}$

Conservative management of colovesical fistula was not attempted in our case series and main mode of treatment was surgery. Multidisciplinary team of urologists and gastro surgeons were involved in the management. Bowel preparation was given on the night prior to the surgery in all cases. Exploratory laparotomy through mid-line incision was electively planned and the first step was to identify site of fistula followed by separation of bowel and bladder. In some cases, because of dense inflammatory and fibrotic reaction separation was not possible leading to partial cystectomy. We have not used frozen section in any of six cases.

Definite management of diverticular disease of colon is required to avoid the recurrence of fistula. Gastro surgical team will decide the limit of resection of bowel. It varied from limited resection to left colectomy. Postoperatively antibiotics judiciously used to avoid minor and major wound sepsis. As majority of pelvic surgery carries the risk of pulmonary embolism, we lost one patient because of it. All five cases were regularly followed up and recurrence of fistula was seen in one case at one year of follow up.
The goal of operative management is to separate and close the involved organs with minimal anatomic disruption and normal long-term function of both bladder and bowel systems. Both single and multistage procedures have been advocated, depending on the clinical circumstances. A one-stage procedure involves removal of the fistula, closure of the involved organs, and primary anastomosis of the bowel after resection of the involved bowel segment. A two-stage approach advocates removal of the fistula, closure of the involved organs and creation of a temporary proximal diverting colostomy. The second stage involves closure of colostomy, Pollard et $\mathrm{al}^{4}$ in their study of 66 cases, in 32 patients single stage resection was done without any significant morbidity and mortality. ${ }^{20,21,22,23,24}$ Nikhil et al ${ }^{25}$ in their case report of colovesical fistula single stage sigmoid resection done and omental flap was interposed between bladder and bowel. Hartmann's procedure is not popular as reversal is difficult due to adhesion formation from previous surgery and pelvic sepsis. ${ }^{26}$ Laparoscopic management of colovesical fistulae has been reported with a relatively high rate of conversion to open repair. ${ }^{27}$ Multistage procedure is done in unprepared bowel, gross contamination, pelvic abscess, radiation injury, advanced malignancy, intestinal obstruction, major medical problem and in advanced age. Nonoperative management is a viable option in selected patients. In nontoxic, minimally symptomatic patients with nonmalignant causes of enterovesical fistulae, a trial of medical therapy including intravenous total parenteral nutrition bowel rest, and antibiotics may be warranted.

\section{CONCLUSIONS}

Colo-vesical fistulae secondary to diverticular disease has shown a rising incidence in Indian population. Pneumaturia is the most common presenting symptom. Diagnosis of diverticular colovesical fistula is challenging and it needs a high index of suspicion in a physician's mind while treating the case of recurrent UTIs or cystitis refractory to antibiotic therapy. CECT abdomen and pelvis with oral, rectal and intravenous contrast is the most valuable diagnostic modality to confirm diverticular CVF. Cystoscopy can be added up to locate the site of fistula and the commonest site of fistula was on left superolateral wall of bladder. Single stage repair without defunctioning colostomy can be effectively performed to cure this entity. Diverticular colovesical fistula management requires multidisciplinary team of surgical specialists, adequate perioperative nutritional and antibiotic care, and meticulous surgical skills including handling of the bowel which can allow an elective one-stage approach.

Financial or Other Competing Interests: None.

\section{REFERENCES}

[1] Leicht W, Thomas C, Thüroff J, et al. Colovesical fistula caused by diverticulitis of the sigmoid colon: diagnosis and treatment. Urologe A 2012;51 (7):971-4. 
[2] Korzenik JR. Case closed? Diverticulitis: epidemiology and fiber. J Clin Gastroenterol 2006;40 Suppl 3:S112-6.

[3] Kang JY, Melville D, Maxwell JD. Epidemiology and management of diverticular disease of the colon. Drugs Aging 2004;21 (4):211-8.

[4] Pollard SG, Macfarlane R, Greatorex R, et al. Colovesical fistula. Ann R Coll Surg Engl 1987;69 (4):163-5.

[5] Melchior S, Cudovic D, Jones J, et al. Diagnosis and surgical management of colovesical fistula due to sigmoid diverticulitis. J Urol 2009;182 (3):978-82.

[6] Niebling M, van Nunspeet L, Zwaving H, et al. Management of colovesical fistulae caused by diverticulitis: 12 years experience in one medical centre. Acta Chir Belg 2013;113 (1):30-4.

[7] Rao PN, Knox R, Barnard RJ, et al. Management of colovesical fistula. Br J Surg 1987;74 (5):362-3.

[8] Sans JV, Teigell JP, Redorta JP, et al. Review of 31 vesico intestinal fistulas: diagnosis and management. Eur Urol 1986;12 (1):21-7.

[9] Pontari MA, McMillen MA, Garvey RH, et al. Diagnosis and treatment of enterovesical fistulae. Am Surg 1992;58(4):258-63.

[10] Chen SS, Chou YH, Tiu CM, et al. Sonographic features of colovesical fistula. J Clin Ultrasound 1990;18 (7):589-91.

[11] Amendola MA, Agha FP, Dent TL, et al. Detection of occult colovesical fistula by the Bourne test. AJR Am J Roentgenol 1984;142 (4):715-8.

[12] Jarrett TW, Vaughan ED. Accuracy of computerized tomography in the diagnosis of colovesicle fistula secondary to diverticular disease. J Urol 1998;153 (1):446.

[13] Zonca P, Jacobi CA, Meyer GP. The current view of surgical treatment of diverticular disease. Rozhl Chir 2009;88 (10):568-76.
[14] Najjar SF, Jamal MK, Savas JF, et al. The spectrum of colovesical fistula and diagnostic paradigm. Am J Surg 2004;188 (5):617-21.

[15] Labs JD, Sarr MG, Fishman EK, et al. Complications of acute diverticulitis of the colon: improved early diagnosis with computerized tomography. Am J Surg 1988;155 (2):331-6.

[16] Morse FP, Dretler SP. Diagnosis and treatment of colovesical fistula. J Urol 1974;111 (1):22-4.

[17] Bourne RB. New aid in the diagnosis of vesicoenteric fistula. J Urol 1964;91:340-2.

[18] Geier GR, Ujiki GT, Shields TW. Colovesical fistula. Arch Surg 1972;105 (2):347-51.

[19] Kwon EO, Armenakas NA, Scharf SC, et al. The poppy seed test for colovesical fistula: big bang, little bucks! J Urol 2008;179 (4):1425-7.

[20] McConnell DB, Sasaki TM, Vetto RM. Experience with colovesical fistula. Am J Surg 1980;140 (1):80-4.

[21] Steele M, Deveney C, Burchell M. Diagnosis and management of colovesical fistulas. Dis Colon Rectum 1979;22 (1):27-30.

[22] Gallagher DM, Russell TR. Surgical management of diverticular disease. Surg Clin North Am 1978;58 (3):56372.

[23] Steier ME, Mitty WF, Nealon TF. Colovesical fistula. J Am Geriatric Soc 1973;21 (12):557-60.

[24] Krco MJ, Malangoni MA, Jacobs SC, et al. Colovesical fistulas. Urology 1984;23 (4):340-2.

[25] Ranjan N, Ahmed A, Rohit K, et al. Colovesical fistulae due to diverticular disease of a sigmoid colon: a case report. UroToday Int J 2013;6 (5):art 61.

[26] Pheils MT. Colonic diverticular disease: colovesical fistula. Dis Colon Rectum 1975;18 (7):560-2.

[27] Joo JS, Agachan F, Wexner SD. Laparoscopic surgery for lower gastrointestinal fistulas. Surg Endosc 1997;11 (2):116-8. 